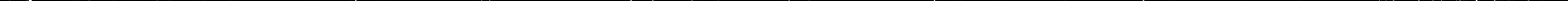


LBL-33236

UC-410

LSGN-120

MODELING AND MEASUREMENT OF THE ALS U5 UNDULATOR END MAGNETIC STRUCTURES

\author{
D. HUMPHRIES, K. HALBACH, E HOYER, \\ B. KINCAID, S. MARKS, and R. SCHLUETER \\ ACCELERATOR and FUSION RESEARCH DIVISION \\ Lawrence Berkeley Laboratory \\ University of California \\ Berkeley, CA 94720
}

MAY 1993

This work was supported by the Director, Office of Energy Research, Office of Basic Energy Sciences, Materials Sciences Division, of the U.S. Department of Energy under Contract No. DE-AC03-76SF00098.

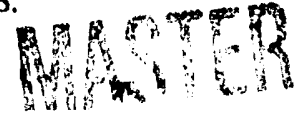




\title{
Modeling and Measurement of the ALS U5 Undulator End Magnetic Structures*
}

\author{
D. Humphries, K. Halbach, E. Hoyer, B. Kincaid, S. Marks, and R. Schlueter \\ Lawrence Berkeley Laboratory, University of California \\ 1 Cyclotron Road, Berkeley, CA 94720 USA
}

\begin{abstract}
The end structures for the ALS U5.0 undulators utilize a system of dual permanent magnet rotors intended to establish gap independent field performance. They may also be used for tuning of the first and second magnetic field integrals of these devices. The behavior of these structures has been studied by means of two dimensional modeling with the POISSON Group of computer codes. A parametric study of the magnetic field distribution and first and second integrals of the fields has been conducted. In parallel, magnetic measurements of the final completed structures have been performed using an automated Hall probe measurement system. Results of the modeling and measurements are compared. Implications for tuning of the ends of the devices within the context of the electron beam parameters of the ALS are discussed.
\end{abstract}

\section{INTRODUCTION}

The first two U5.0 undulators for the Advanced Light Source (ALS) [1] are hybrid permanent magnet structures which have mirror symmetric end structures. The engineering design of these end structures was based upon a truncation theory [2] which indicated a means of compact termination of the structure and minimization of $1 \mathrm{st}$ and 2 nd field integral effects on the electron beam of the ALS.

\section{2-D NUMERICAL MODEL}

To model the U5.0 end structures, a 2-D numerical nonlinear magnetostatic problem was constructed. Figure 1 illustrates the geometry of the model and the vector potential solution (field lines) for a particular case. The upper half of the last three periods of the magnetic structure are included in the model. The left boundary condition is Dirichlet $(\vec{A}=0)$

while the remaining three are Neumann $(\nabla \vec{A} \cdot \hat{n}=0)$. The upper and right boundaries are remote and not shown in the figure. A magnetic shunt is shown attached to pole 0 at the far right. This shunt is connected to the upper Neumann boundary and provides a flux return path through an implied infinite permeability region formed by the upper and right Neumann boundaries.

The eight regions with orientation arrows represent the $\mathrm{Nd}-\mathrm{Fe}-\mathrm{B}$ permanent magnet material which energizes the structure. The six rectangular regions are fixed material while the two octagonal regions represent elements that can be rotated. Seven pole regions are shown with the far left pole split at the Dirichlet boundary symmetry plane.

*This work was supported by the Director, Office of Energy Research, Office of Basic Energy Sciences, Materials Sciences Division of the U.S. Department of Energy, under Contract No. DE-AC03-76SF00098.
The geometry shown, in conjunction with the described boundary conditions, gives calculated fields which are equivalent to those of a complete upper and lower, 26 pole, 2 $\mathrm{D}$ device.

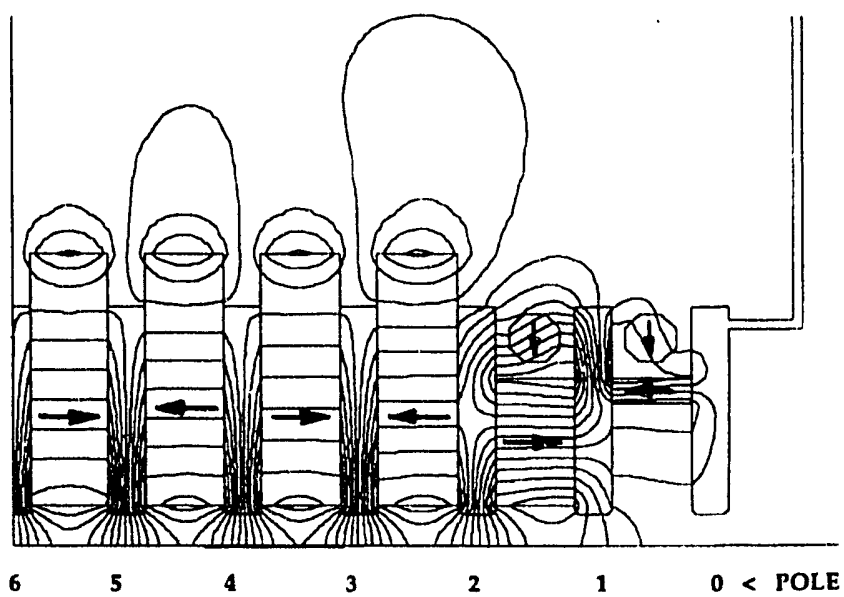

Figure 1: 2-D PANDIRA Model

\section{CALCULATED FIELDS}

PANDIRA a member of the POISSON Group [3] of computer codes, was used to solve this model. PANDIRA is able to model 'analytic' anisotropic material with arbitrary orientations via simple B-H intercepts and easy axis orientation angles. This is particularly helpful in this application since it allows rotor orientation to be varied by changing a single number in the input parameter list.

The permanent magnet material used for these devices is $\mathrm{Nd}-\mathrm{Fe}-\mathrm{B}$. This material was modeled using a linear B-H curve with intercepts of $B_{r}=11000 G$ and $H_{c}=-10700$ Oe. The pole material is vanadium permendur and was modeled using a nonlinear B-H curve.

PANDIRA solves Poisson's equation directly for the vector potential $(\vec{A})$ on an irregular triangular mesh. The mesh for this model contains approximately 15000 intersection points with high density in the regions of interest, e.g. the gap. The code then applies a differentiated interpolating polynomial to the nodes of the problem to calculate the $\vec{B}$ fields. Thus we have $\vec{B}$ and $\int \vec{B} d z$ immediately from PANDIRA.

To further extract information from the model, the calculated $B_{y}$ and vector potential information was transferred to a graphical spreadsheet. The 2 nd integral of $B_{y}$ on the mid plane was then calculated and a 'progressive integral signature' was generated which is shown in figure 2 .

On this graph, $z=0 \mathrm{~cm}$ is the left Dirichlet boundary of the PANDIRA model. The seventh and last pole (pole 0 ) is at $\mathrm{z}=$ $15 \mathrm{~cm}$. The solid line on the graph is $B_{y}$ calculated on the mid 
plane of the device. The line shown in long dashes is th -irst integral of $B_{y}$ i.e. the vector potential.

The line shown in short dashes is the second integral of $B_{y}$ which defines a path proportional to the trajectory (rotated 90 deg) of a particle traveling through the mid plane field. The portion of this path from $\mathrm{z}=0$ to $\mathrm{z}=2.5 \mathrm{~cm}$ of this trajectory indicates the relative amplitude of the transverse particle oscillation through the periodic section of the device. The deviation of the remaining part of the trajectory can thus be compared to this oscillation amplitude to see the significance of tuning effects in the model.

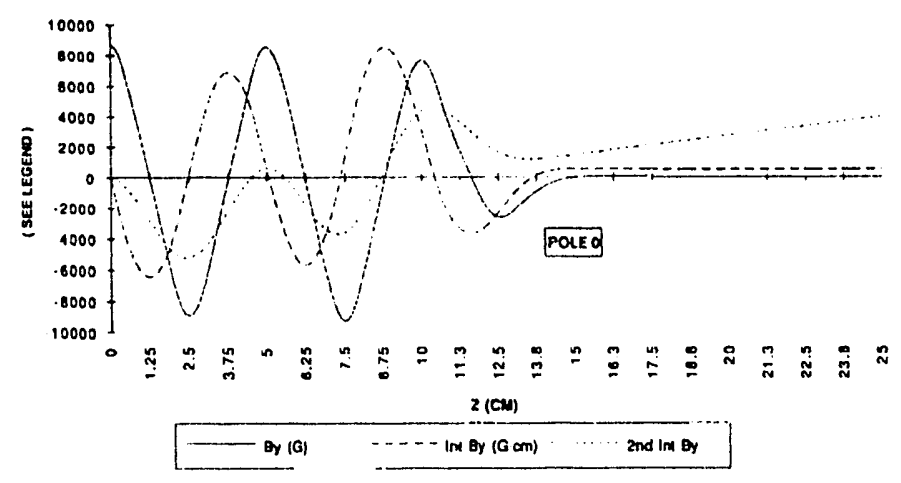

Figure 2: Progressive Integral Signature

If we examine the first integral curve in this graph we see that it takes on a positive constant value for $z>15 \mathrm{~cm}$ (where $B_{y}=0$ ). This implies a net integrated steering field produced within this end configuration. Correspondingly, we see the effect on the trajectory curve as it diverges linearly from the $\mathrm{z}=15 \mathrm{~cm}$ point on the graph.

\section{COMPARISION TO MEASUREMENTS}

In parallel with the construction of the U5.0 undulators, a state-of-the-art automated magnetic measurement system [4] was developed to measure and characterize the devices. This system is able to perform high precision field mapping using Hall probes. The measurement data are processed using spline interpolation to generate a smooth function which is evaluated at 6000 points.

To compare the model results with the measured results, the ends of the data sets were extracted and re-interpolated to produce field values at the exact locations of the calculated values for the 2-D PANDIRA model.

Figure 3 shows a comparison of the calculated $B_{y}$ fields from the 2-D model and those measured in the actual device. The 2-D model was uriginally scaled to produce a $B_{0}$ value of .87 tesla. For this comparison the 2-D fields were scaled to give exact equivalence to the measured $B_{0}(.9$ tesla) at $\mathrm{z}=0 \mathrm{~cm}$. This gives a picture of the divergence of the two results moving through the end region of the magnetic structure. As shown in the graph, the agreement between the two results is good with a discrepancy of only a few percent near the last two poles of the end structure.

The most significant disagreement between the model and the real device occurs in the gap dependency of the integrated dipole from the ends. Figure 4 shows measured results for the integrated dipole as a function of gap. A large increase of approximately $900 \mathrm{G}$-cm occurs in the integral of $B_{y}$ as the gap approaches $14 \mathrm{~mm}$.

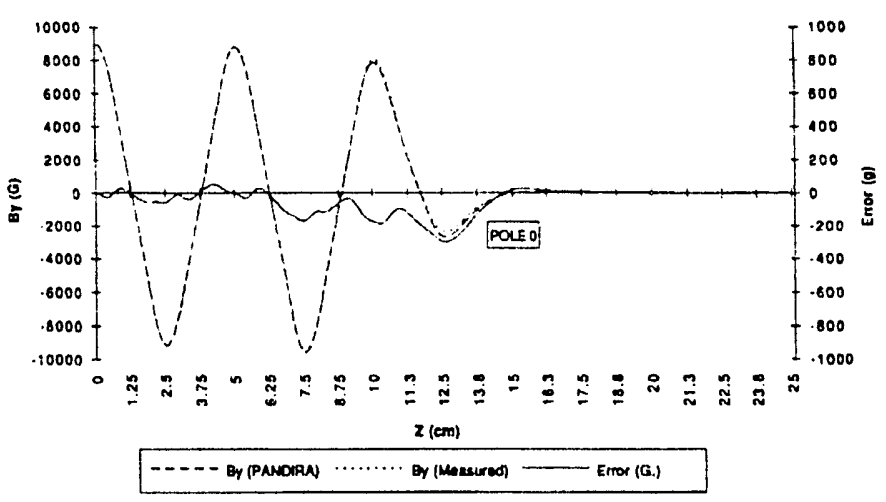

Figure 3: Calculated vs Measured $B_{y}$

The 2-D model manifests no such increase, even when the gap of the model is closed to $10 \mathrm{~mm}$. The model shows a maximum variation over this gap range of only $100 \mathrm{G}-\mathrm{cm}$. This behavior was not predicted by the original theory and initial conjecture is that it is likely to be related to a 3-dimensional effect which occurs at small gaps.

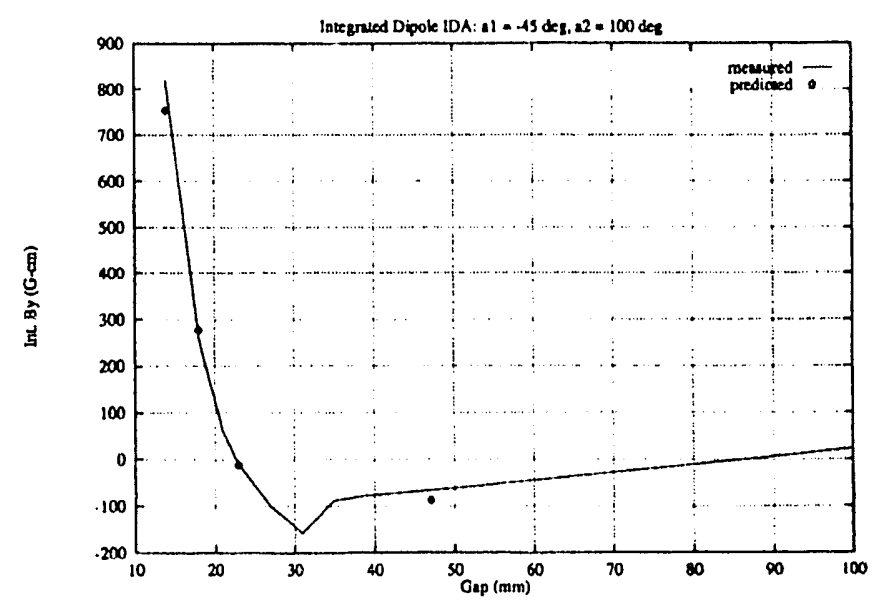

Figure 4: Measured Integrated Dipole

\section{ROTOR EFFECTS}

The primary effect of the rotors is to vary the scalar potential of the adjacent poles and thus to effect a change in the field distribution at the mid plane. Maximum effect is nbtained for rotors oriented horizontally at approximately 0 or 180 degrees. The field integral at the mid plane is non-zero for rotor orientation combinations which cause unequal pole potential sums above and below the midplane.

The undulator magnetic structures for these devices are surrounded on three sides by steel backing beams which act to shield the overall structure from ambient fields. Flux shunts connect the last pole at each end of each magnetic structure to the backing beam ends. The upper and lower backing beams are magnetically connected via hinged yokes which allow a low reluctance path to be maintained during opening and closing of the magnet gap. In the 2-D model, these backing 
beams and return yokes are represented by the upper and right hand Neumann boundary. A complete flux return path is thus established from the upper magnetic structure to the lower magnetic structure to carry any unbalanced error flux or induced flux from the rotors which crosses the air gap between the poles.

In addition to the primary rotor induced flux carried through the shunts, there is a secondary induced flux effect from direct magnetic linkage of the poles to the backing beam through the air. This effect is maximum for approximately vertical (90 and 270 degree) rotor orientations.

This combination of primary and secondary effects leads to an analytic characterization of the rotors in terms of their orientation angles and the resulting net end steering effect or interral of $B_{y}$ at the mid plane.

Letting $\alpha_{1}$ be the orientation of the inner or left rotor as shown in figure $I$ and $\alpha_{2}$ be the orientation of the outer or right rotor then $I_{S}$ where $I_{s}=\int B_{y} d z$ can be expressed as follows:

$$
\begin{aligned}
& I_{s}=c_{0}+c_{1} \cos \left(\alpha_{1}\right)+c_{2} \sin \left(\alpha_{1}\right) \\
& +c_{3} \cos \left(\alpha_{2}\right)+c_{4} \sin \left(\alpha_{2}\right)
\end{aligned}
$$

The $c_{n}$ are obtained in each case by calculating or measuring the integral of $B_{y}$ for multiple rotor orientations and performing a simple matrix calculation.

Figure 5 is a graphical representation of this function evaluated for the 2D model case. This graph shows that the outer rotor has approximately twice the effect of the inner rotor. Also shown is a slight asymmetry along both the $\alpha_{1}$ and $\alpha_{2}$ axes resulting from the sine terms in equation (1) above.

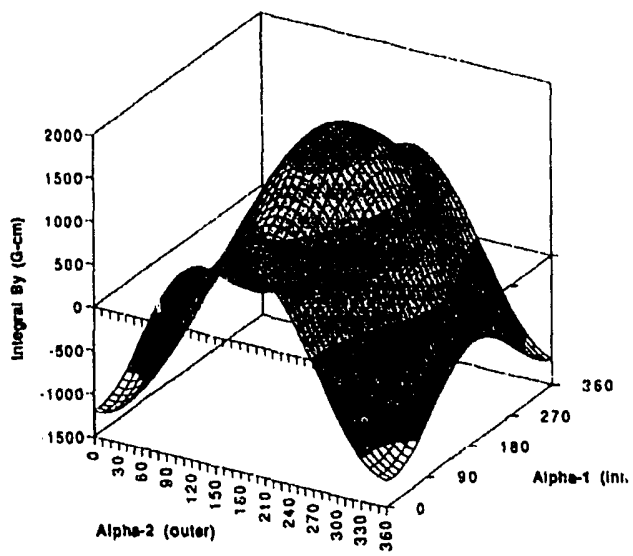

$$
\left[\begin{array}{l}
1500 \cdot 2000 \\
1000.1500 \\
500 \cdot 1000 \\
0.500 \\
.500 .0 \\
.1000 \cdot .500 \\
\square .1500 \cdot .1000
\end{array}\right]
$$

Figure 5: Rotor Effect on $\int B_{y} d z$ for 2-D Case

The corresponding function was calculated for the measured case which yielded a similar contour but with an amplitude approximately .6 times that of the 2-D model. This discrepancy is attributable to some combination of inaccurate modeling of the flux return path through the backing beam and, to a lesser degree, errors in the 2-D equivalent rotor volume in the PANDIRA model.

To make a meaningfui comparison of the two functions, they were both zero shifted by averaging. The 2-D contour was then normalized to peak amplitude of the measured contour and a difference contour was obtained. This is shown in Figure 6 and indicates relatively good qualitative agreement between the two cases with a standard deviation of the error of approximately $44 \mathrm{G}-\mathrm{cm}$ or less than $5 \%$ of the peak measured rotor effect on $\int B_{y} d z$.

Qualitative Error in Integral By - PANDIRA ys Moasured

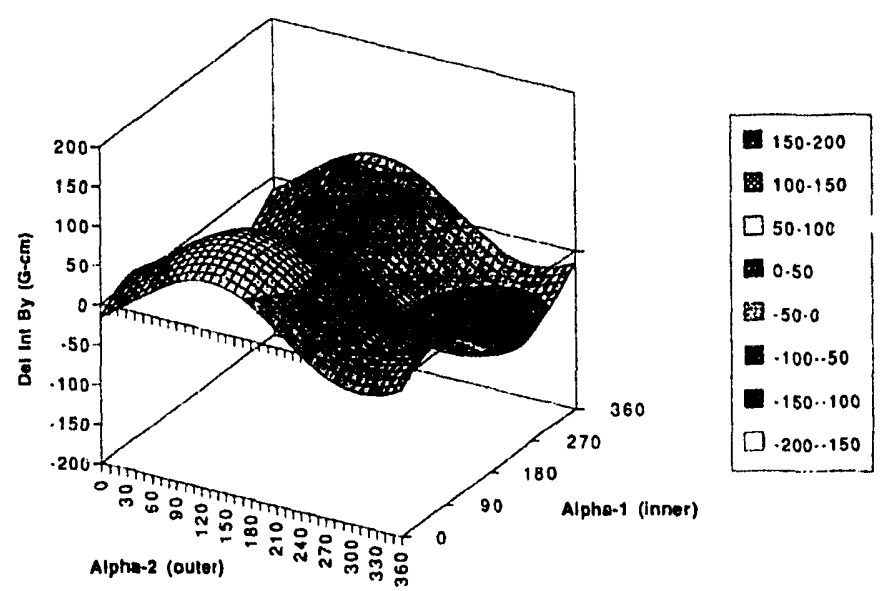

Figure 6: Rotor Effect Difference Contour, 2-D vs Measured

\section{CONCLUSIONS}

In spite of certain areas of disagreement, 2-D modeling has proven to be a useful and cost effective tool for the understanding and design of the complex end structures of the U5.0 undulators. It has yielded good qualitative agreement with the actual measured de vices in most areas. It has also pointed to subtle behaviors which were not predicted by the original theory. We plan to utilize this modeling technique to refine the end designs of future insertion devices for the ALS.

\section{REFERENCES}

[1] E. Hoyer, et al, "First Undulators for the Advanced Light Source", IEEE PAC (May 1993).

[2] "U5.0 Undulator Conceptual Design Report", LBL PUB-5256, Sect 5.2.2, (Nov. 1989).

[3] "POISSON/SUPERFISH Reference Manua!". LANL LA-UR-87-126, (Jan. 1987).

[4] S. Marks, et al, "ALS Insertion Device Magnetic Measurements", IEEE PAC (May 1993). 


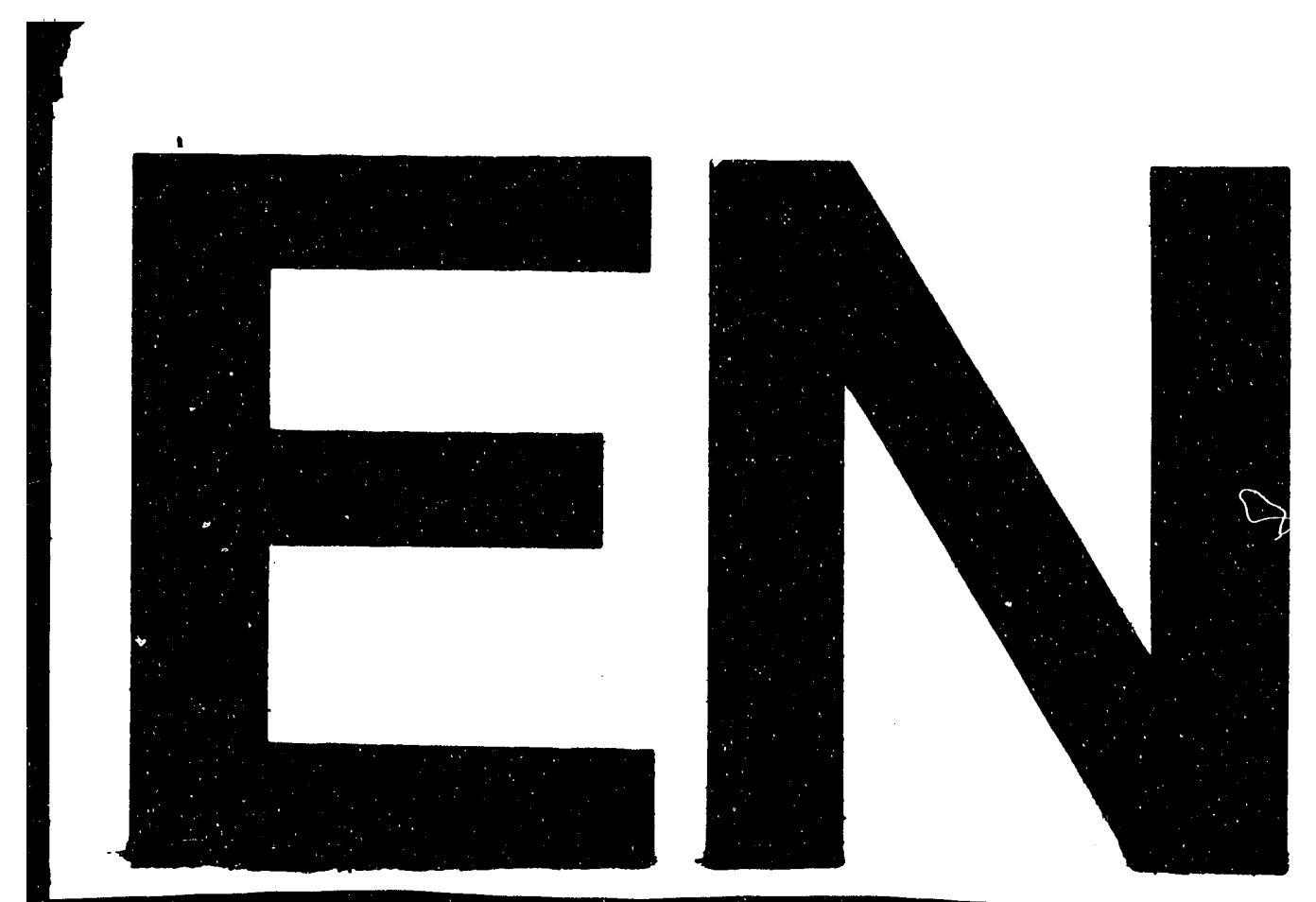

$\nabla$
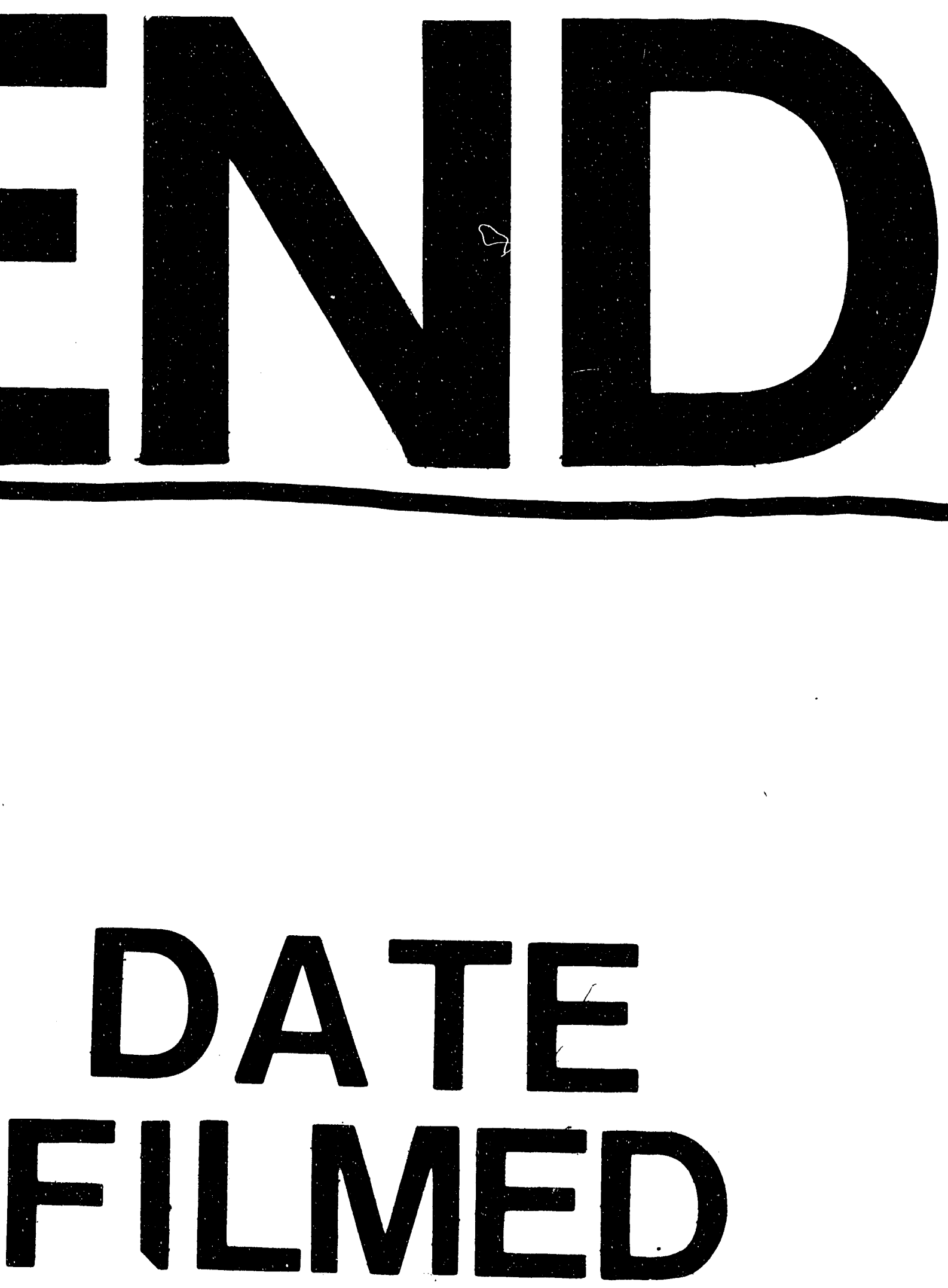

$9 / 13 / 93$ 


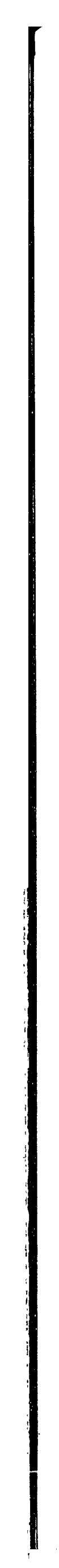

I

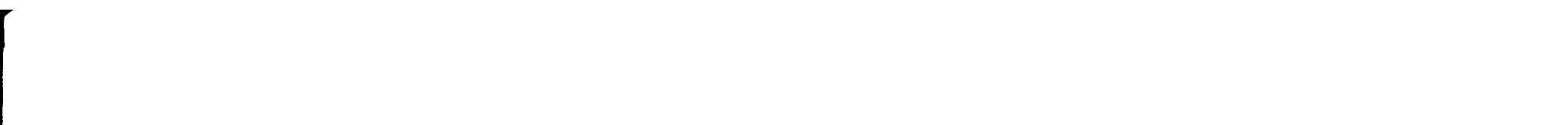

.$$
\text { . }
$$
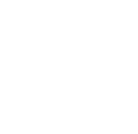